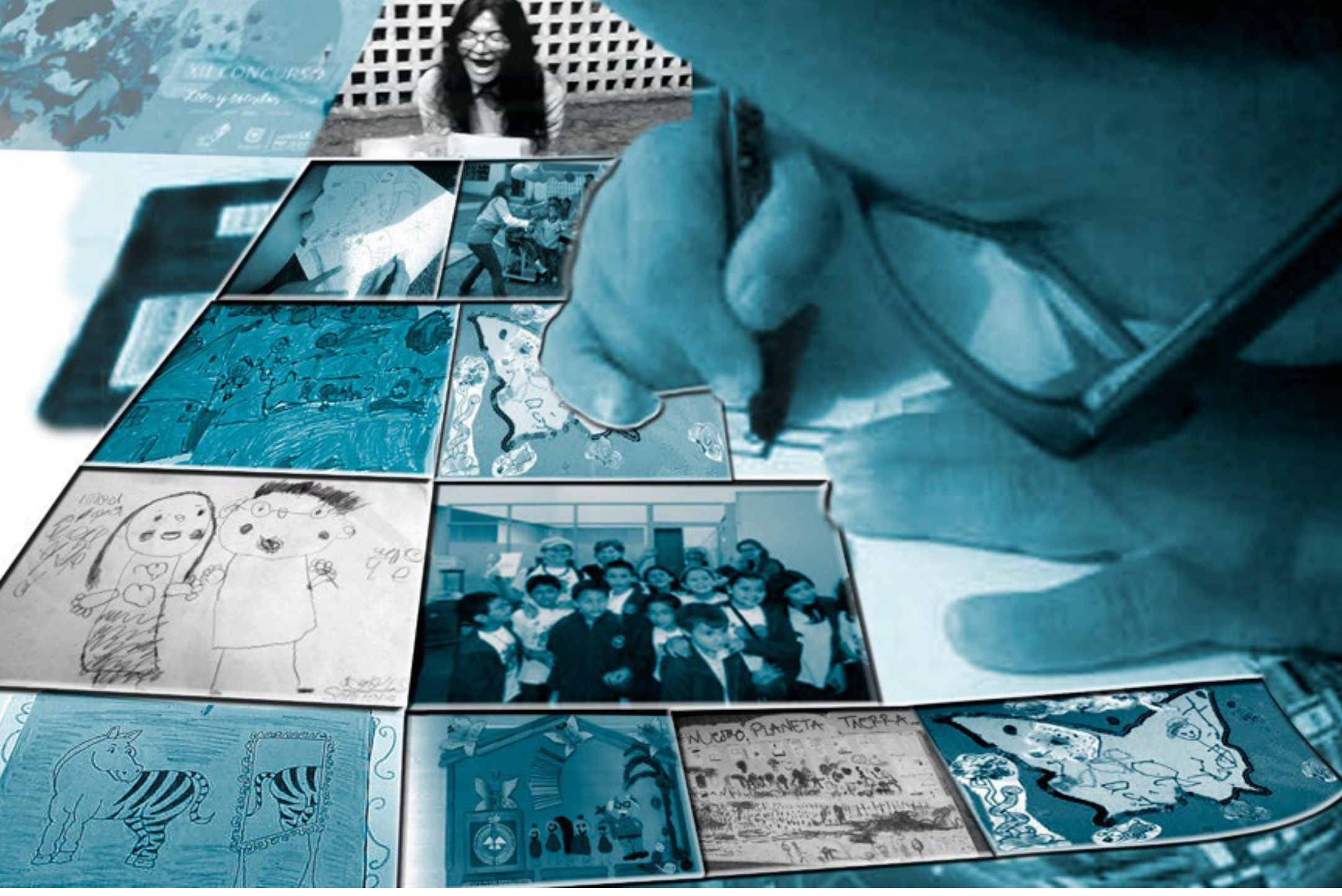

\title{
Apapaches: leer, acariciar el alma con los libros. Una micro política educativa en el aula, enmarcada en la felicidad y la expresión
}

Apapaches: read, caress the soul with books. A micro-educational policy in the classroom, framed in happiness and expression

Apapaches: leer, acariciar a alma com os libros. Uma política micro educativa na aula, enmarcada na felicidade e na expressão

Blanca Lilia Medina 


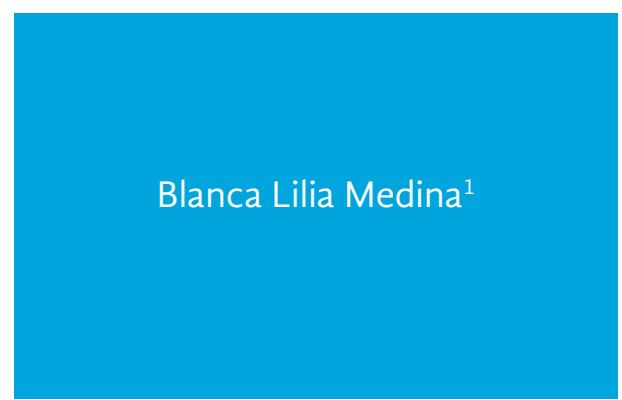

Docente, curso Jardín, Colegio Gabriel Betancourt Mejía IED, Localidad octava de Kennedy, Bogotá. Magister en Estructuras y procesos del aprendizaje, Universidad Externado de Colombia; correo electrónico: blanlilia@yahoo.com

Fecha de recepción: 27 de noviembre de 2017 / Fecha de aprobación: 30 de marzo de 2018

\section{Resumen}

El siguiente artículo presenta el resultado de la experiencia pedagógica "Apapaches", que desde 2007 surge como una micro política educativa en el Colegio Gabriel Betancourt Mejía IED, para fortalecer la expresión oral, escrita y la construcción de confianza en sí mismo en los estudiantes de educación inicial; su principio pedagógico es la felicidad de los niños, razón por la cual el propósito educativo está enmarcado por el juego, el arte y la imaginación, como lenguajes entrañables de la infancia.

Palabras clave: Infancia, lectura, expresión, desarrollo, juego, micro política educativa.

\section{Abstract}

The following article presents the result of the pedagogical experience "Apapaches", which since 2007 emerges as a micro-educational policy at the Gabriel Betancourt Mejía IED School, to strengthen the oral and written expression as well as the construction of selfconfidence in the students of Initial education; Its pedagogical principle is the happiness of children, which is why the educational purpose is framed in play, art and imagination, as endearing languages of childhood.

Keywords: Childhood, reading, expression, development, game, micro educational policy.

\section{Resumo}

$\mathrm{O}$ artigo a seguir apresenta o resultado da experiência pedagógica "Apapaches", que desde 2007 surge como uma política de microeducação na Escola de IED Gabriel Betancourt Mejía, para fortalecer a expressão oral, escrita e a construção da autoconfiança nos estudantes de Educação inicial; Seu princípio pedagógico é a felicidade das crianças, e é por isso que o propósito educativo é enquadrado pela brincadeira, a arte e a imaginação, como linguagens cativantes da infância.

Palavras-chave: Infância, leitura, expressão, desenvolvimento, educação, política educacional. 


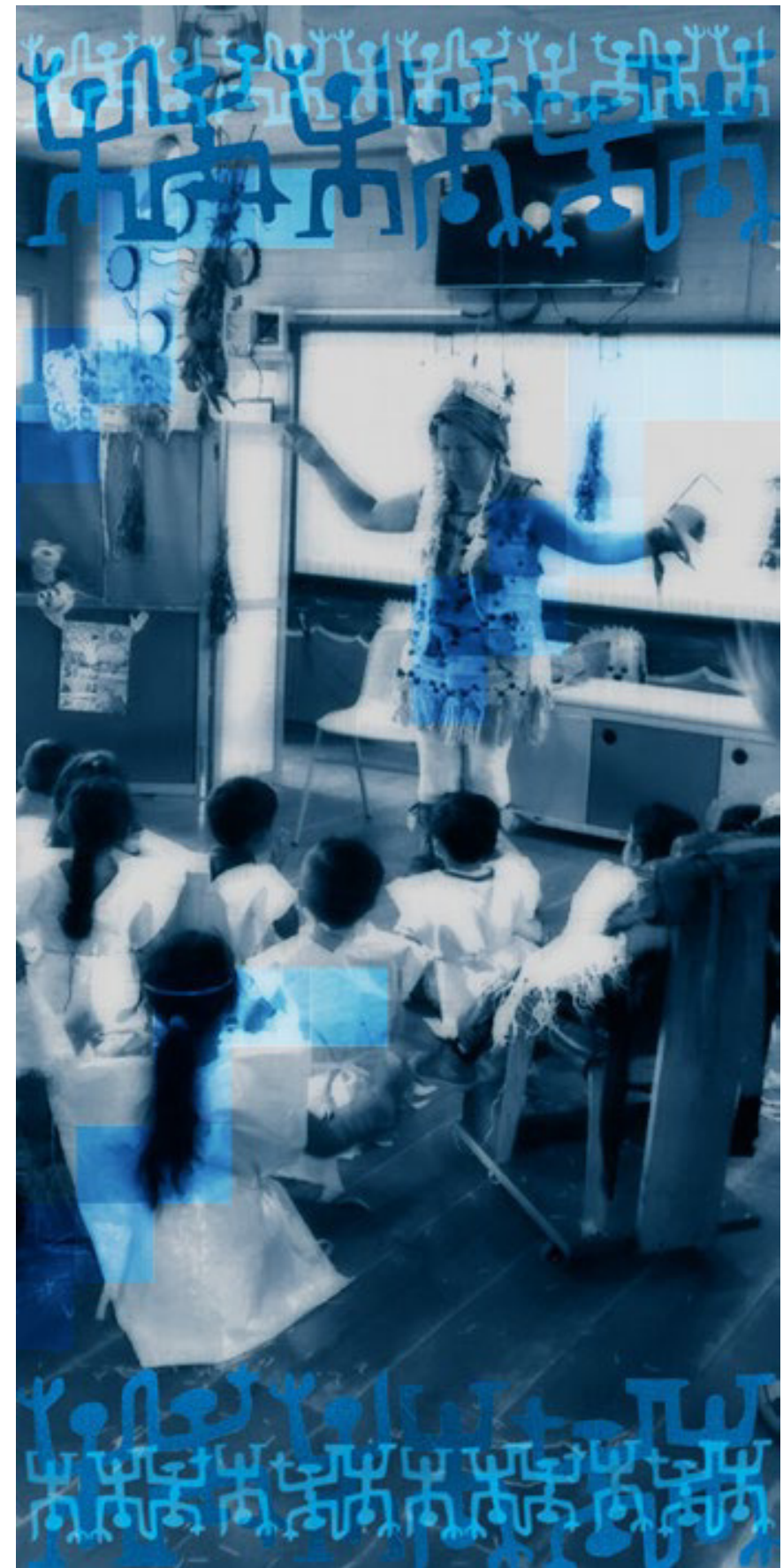

\section{Punto de partida, a manera de prefacio}

De los diversos instrumentos del hombre, el más asombroso es, sin duda, el libro. Los demás son extensiones de su cuerpo.

El microscopio, el telescopio son extensiones de su vista; el teléfono es extensión de la voz; luego tenemos el arado y la espada, extensiones del brazo. Pero el libro es otra cosa: el libro es una extensión de la memoria y la imaginación. Jorge Luis Borges

n Colombia, el Ministerio de Educación Nacional y la Secretaría de Educación del Distrito, (SED) han hecho énfasis, durante los últimos años, en las prácticas de aula y en la manera como cada docente asume la enseñanza de la lectura y la escritura en su contexto educativo, para, desde las prácticas educativas, enfrentar los retos sociales, económicos y culturales del mundo globalizado; por este motivo se ha hecho necesario que los planes de estudio y el currículo adquieran la forma y características propias de cada contexto, para actuar desde allí y "hacer" educación.

Partiendo de tal perspectiva, desde hace diez años se adelanta la propuesta "Apapaches” en el Colegio Gabriel Betancourt Mejía, ubicado en el barrio Tintal, localidad octava de Kennedy, Bogotá. En principio se constituyó como proyecto de aula con estudiantes de los cursos de transición, pre jardín y jardín; sin embargo, gracias al impacto logrado en la población estudiantil y a los aportes del proyecto al modelo educativo de la institución, la labor se extendió al tiempo extraescolar, donde participan actualmente estudiantes de primaria y bachillerato.

El club de lectura cuenta con la participación de los estudiantes ganadores del concurso "Bogotá una historia esperando ser contada. Concurso leer y escribir” (2016-217) (2017-2018), de la Secretaría de Educación, y de un alumno ganador del primer puesto en reseña "Que la violencia no se te pegue, colegios con respeto y equidad para mujeres, niñas y niños" (2013-2014), con el título "El libro de madera"2. Apapaches ha implementado didácticas atractivas

Alcaldía mayor de Bogotá. Secretaría de Educación del Distrito: "Porque leer es volar, Bogotá una historia esperando ser contada, 2017. Concurso leer y escribir". Primer puesto, reseña y cuento, segundo puesto, ilustración y reseña: "Que la violencia no se te pegue, colegios con respeto y equidad para mujeres, niñas y niños", primer puesto, reseña. 
para que el estudiante incursione en el mundo de la lectura desde la participación, a partir de estrategias como "El contrato lector", donde expresa abiertamente su deseo y firma, junto a sus padres, el compromiso amistoso con la lectura.

Apapaches se apoya en los aportes de la pedagogía de proyectos, desde la psicopedagogía, que busca demostrar que la actividad de quien aprende es determinante en la construcción de un saber operatorio, y que este sujeto, el cual nunca está aislado, actúa en interacción permanente con los medios de su entorno. El proyecto moviliza la cotidianidad escolar en tanto transformación pedagógica, en un aula que desafía a la institución educativa a repensar y replantear sus prácticas de enseñanza y aprendizaje; se ha convertido en alternativa para el fomento y promoción de la lectura, la oralidad y la escritura, pues permea las prácticas pedagógicas, fomenta la participación y moviliza los procesos reflexivos en torno a las concepciones y hábitos del aula.

El trabajo realizado trasciende las fronteras del aula, sin dejar de lado las cartas de navegación que han conducido la implementación de didácticas desde el horizonte de la diversidad, ellas son: nuestro PEI gabrielista "Formación de ciudadanos dialógicos y humanistas con conciencia social", el Plan Nacional de Lectura y Escritura "Leer es mi cuento" (MEN), los Derechos básicos de aprendizaje (MEN), el Lineamiento pedagógico y curricular para la Educación Inicial en el Distrito, y las estrategias didácticas, a través de la incorporación de la escritura, la lectura y la oralidad en primer ciclo (SED).

Acudir a la memoria histórico pedagógica de Apapaches permite ubicar en una línea del tiempo todos los aportes que han permitido transitar, desde las prácticas tradicionales para el aprendizaje de la lectura y escritura, hasta una visión cultural y social que permite el intercambio de saberes, políticas de debate educativo y didácticas atractivas y efectivas para el aprendizaje de la lectura, la escritura y la oralidad; especialmente desde la intervención en el desarrollo infantil, reconociendo que cada niño y niña que asiste al contexto educativo habita una cultura, una naturaleza diversa que configura su existencia y subjetividad.

Así se ha logrado posicionar la singularidad como concepto de infancia y movilizar las transformaciones pedagógicas, sociales y culturales que, a manera de micro políticas de aula, inciden y permean las concepciones, saberes y prácticas de nuestro contexto educativo.

Por lo tanto, para quienes trabajan el currículo y la incidencia de las políticas educativas en las transformaciones sociales, resulta atractivo conocer la propuesta Apapaches, que incide en la vida escolar de estudiantes de educación inicial, primaria e incluso en bachillerato, padres de familia, bibliotecarias, docentes y directivos docentes, como la coordinadora y la orientadora de primaria, quienes en tiempo extraescolar se suman a esta aventura, contribuyendo a la construcción de la primera comunidad lectora y escritora gabrielista: El Club de lectura Apapaches ${ }^{3}$. En síntesis, las prácticas de lectura, escritura y oralidad se dan desde el lenguaje, un terreno fértil para la vida colectiva y el tejido social y humano.

El presente texto es justamente resultado de los procesos, encuentros y desencuentros transitados en esta experiencia pedagógica que se construye y reconstruye día a día, en la interacción con los estudiantes y la búsqueda de nuevas y mejores prácticas para la enseñanza y el aprendizaje.

\section{Actuando en contexto}

Los libros son posibilitadores del encuentro, de la comprensión de lo humano, de la construcción de sentido de vida, que es lo que se trabaja en la escuela [...] Lo que estamos haciendo es amasar ese material humano que es el que va a nutrir la sociedad [...] Los libros y la lectura no son solo conocimiento académico, sino que son vehículos para reencontrarnos, reconciliarnos y comprendernos.

Teresa de Jesús Sierra Jaime, coordinadora G.B.M.

El Colegio Gabriel Betancourt Mejía enfrenta problemas de bajas tasas de lectura y escritura en los niveles de preescolar, básica y media; los docentes buscan responder a la apatía estudiantil frente a estas áreas. Para atender la situación, a nivel institucional se han intentado estrategias como: impulsar el trabajo cooperativo; replantear el PEI; dotar de libros la biblioteca; reforzar tareas con orientación escolar; reflexionar acerca de procesos innovadores como el Método Singapur (comprensión lectora en la resolución de problemas matemáticos) o el método Filadelfia, sobre lectura temprana; y construir, desde la coordinación académica de primaria, proyectos para educación inicial como Puro Cuento ${ }^{4}$, iniciativa que acercó los horizontes conceptuales del lenguaje. Como institución educativa la tenemos clara: ¡Necesitamos mejorar las didácticas para la enseñanza y aprendizaje de la lectura y la escritura!

Sin embargo, esfuerzos y buenas intenciones nunca serán suficientes para alcanzar los resultados esperados, si no cerramos en nuestras aulas la brecha entre experiencia estética de la lectura y su mecanización. ¡El desafío es reflexionar sobre nuestros enfoques y metodologías, construir las herramientas pedagógicas

\footnotetext{
Al respecto, es posible consultar: apapachesgbm.blogspot.com.co

Desarrollado en 2013 por Teresa de Jesús Sierra Jaime y la Coordinación Académica del colegio, jornada mañana, sede A.
} 
necesarias para enfrentar, sin miedo ni engaños, las didácticas "perversas" que se han apoderado de nuestra práctica para enseñar la lectura y escritura!, las mismas que han encerrado la lectura en "cartillas" que excluyen las transformaciones actuales del sistema de comunicación, en el cual los medios audiovisuales han situado la imagen en un lugar privilegiado.

Desde los métodos tradicionales nuestros niños y niñas repiten términos pueriles, que no implican ninguna gracia o encanto para su mundo, exiliando la palabra escrita de su campo social y cultural, atrapándola en los pizarrones, cuadernos de planas y transcripciones de libros, lejos de su corazón, de sus mentes. Tales didácticas mecánicas y repetitivas les prohíben el desarrollo de su imaginación como práctica del ejercicio de ciudadanía a través de la escritura, negando todo el aprendizaje que se deriva de su vida social. La escritura es importante en la escuela porque es importante fuera de ella, no a la inversa; esta perspectiva es la que impulsa la propuesta Apapaches, que busca alcanzar los siguientes propósitos pedagógicos:
1. Diseñar un ambiente de aprendizaje cooperativo desde los medios digitales, para la enseñanza de la lectura, la escritura y la oralidad, buscando estrechar la comunicación entre la familia, los estudiantes y el currículo.

2. Construir estrategias didácticas contextualizadas que permitan a los estudiantes recrear el gusto por la lectura y desarrollar habilidades comunicativas orales y escritas.

3. Impulsar en los estudiantes la expresión oral, escrita y la construcción de confianza en sí mismos, a través de la publicación de sus producciones y la puesta en escena de sus habilidades artísticas y comunicativas.

4. Transformar las prácticas tradicionales de lectura, escritura y oralidad en espacios de inserción cultural y de interacción humana.

El siguiente gráfico presenta una relación del entramado pedagógico, las didácticas y los propósitos de la experiencia.

Gráfico 1. Relación entre didáctica, propósitos y pedagogía

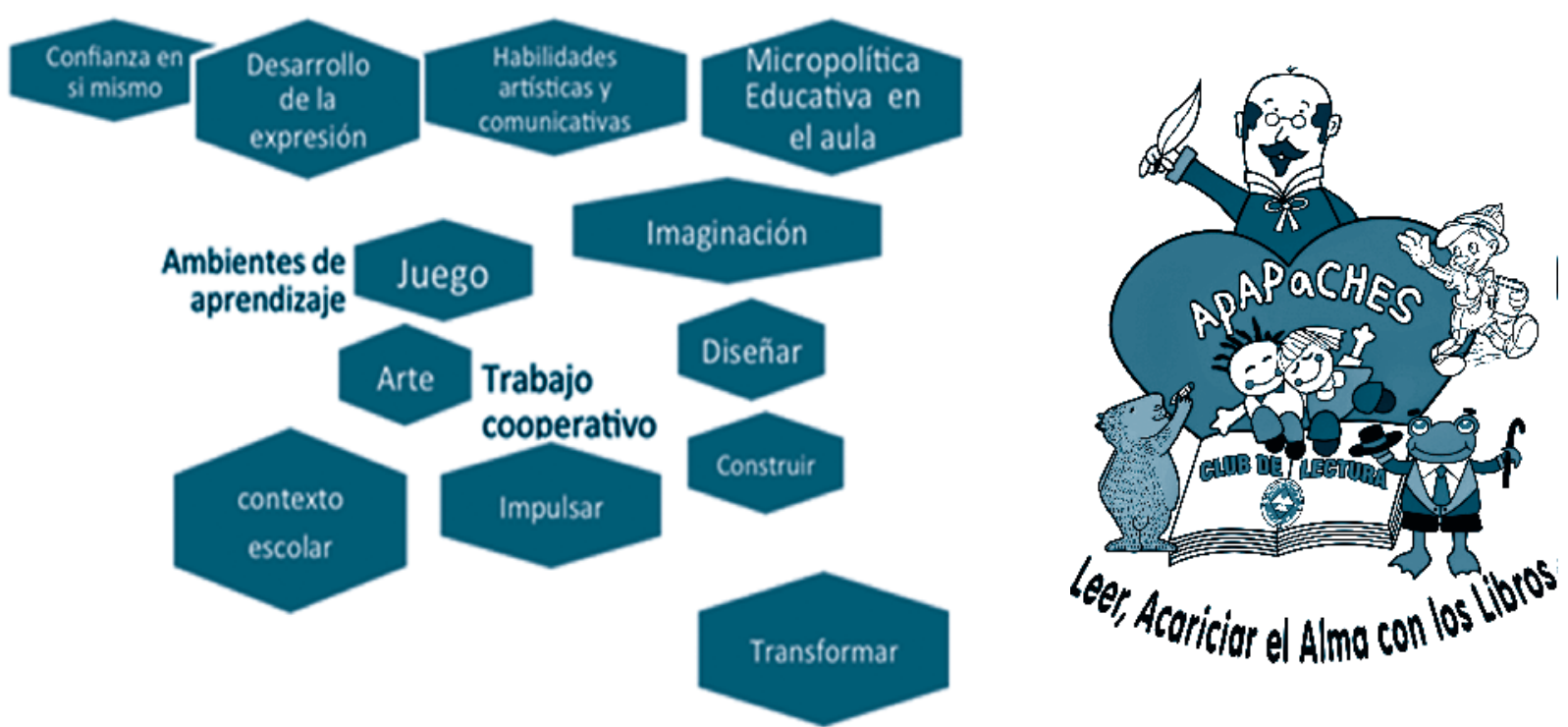

INFANCIA

Nota. Elaborado por la autora 


\section{La escenografía: Sustento pedagógico}

Apapaches tiene como principio pedagógico la felicidad, su propósito es impulsar en los estudiantes la expresión oral, escrita y la construcción de confianza en sí mismos; da un lugar fundamental al juego, el arte y la imaginación, no solo porque son sistemas simbólicos de representación y lenguajes entrañables de la infancia, sino porque median en el fortalecimiento de las dimensiones del desarrollo infantil: personal-social, corporal, comunicativa, artística y cognitiva; las cuales permiten entenderlo, como lo hace la SED, en tanto entramado biológico, psicológico, social, cultural e histórico que fortalece y trabaja las comprensiones, saberes, sentimientos, capacidades y habilidades básicas para la vida del ser humano, la construcción de sí mismo y del mundo exterior.

Pero, ¿cómo convergen cada uno de los elementos propuestos en el currículo con la experiencia pedagógica Apapaches? La respuesta no se resuelve sino en el aula misma, cuando se desarrolla un diálogo entre el currículo y los propósitos pedagógicos de la propuesta Apapaches.

Los seres humanos nacen con la capacidad fisiológica de emitir sonidos y de articularlos, es decir, de hablar, y con la capacidad intelectual de aprender a hacerlo. En tal sentido, Chomsky (1928) sostiene que esta "propiedad de la especie" presenta escasa variación entre los seres humanos y no se repite en otros seres biológicos; así, el origen y uso del lenguaje es reservado para la comunicación humana y su exclusividad se da en tanto que el ser humano convierte los estímulos externos en material significante que conduce hacia una interpretación (Avendaño y Perrone, 2012, p. 15).

[...] La ciencia que se ha constituido en torno de los hechos de la lengua ha pasado por tres fases sucesivas: la gramática, la filología y la gramática comparada.

[...] La lingüística tiene que diferenciarse cuidadosamente de la etnografía y la pre-historia, donde el lenguaje no interviene más que a título de documento; tiene que distinguirse también de la antropología, que no estudia al hombre más que desde el punto de vista de la especie, mientras que el lenguaje es un hecho social [...] El estudio de la gramática inaugurado por los griegos, continuado especialmente por los franceses, está fundado en la lógica y desprovisto de toda visión científica y desinteresada de la lengua misma; lo que la gramática se propone únicamente es dar reglas para distinguir las formas correctas de las formas incorrectas; es una disciplina normativa, muy alejada de la pura observación, y su punto de vista es necesariamente estrecho (Saussure, 1916, p. 43).
Por su parte, el "signo lingüístico" puede ser definido como cada una de las palabras que componen una lengua; para comunicar, los signos lingüísticos de una lengua integran un significante a un significado. Una vez elegido el modelo y establecido el vínculo entre significante y significado, ni el hablante individual ni el grupo en su totalidad pueden romperlo. No obstante, es imprescindible considerar que estas capacidades adquieren sentido solo si hay un campo social, un sistema de signos común para todos los miembros del grupo.

En esta medida, Apapaches une sus esfuerzos pedagógicos a una "psicología cultural” que se ocupa esencialmente del significado.

[...] El estudio de la mente humana es tan difícil, se encuentra tan inmerso en el dilema de ser a la vez el objeto y el sujeto de su propio estudio, no puede limitar sus indagaciones a las formas de pensamiento que se desarrollaron a partir de la física de ayer. La tarea es tan apremiantemente importante que merece la rica variedad de inteligencia y perspicacia que seamos capaces de aportar a la comprensión de lo que el hombre piensa de su mundo, de sus congéneres y de sí mismo. Este es el espíritu con el que hemos de avanzar [...] Un punto de partida [es la] revolución cognitiva [...] cuyo objetivo era recuperar la "mente" en las ciencias humanas después de un prolongado y frío invierno de objetivismo. La ciencia cognitiva se encuentra sin duda entre las acciones más cotizadas de la bolsa académica [...] Se basa en un enfoque más interpretativo del conocimiento, cuyo centro de interés es la "construcción de significados" (Bruner, 1999, p. 14).

De lo anterior es posible destacar una preocupación: ¿En nuestras aulas se lee por gusto o para darle gusto al profesor? La educación, tal como existe en la actualidad, reprime el pensamiento, transmite datos, conocimientos, saberes y resultados de procesos que otros pensaron, pero no enseña ni permite pensar, en ella hay gran incomunicación, y el tengo que llegar a saber "algo" es resultado de un proceso que no se enseña. Saber significa entonces simplemente repetir ${ }^{5}$.

\section{Luces, cámara, acción. Las estrategias}

Con el propósito de aprovechar los beneficios del aprendizaje cooperativo, para el proyecto se ha puesto en marcha una micro política educativa en el aula, con estrategias y acciones pedagógicas que han permitido alcanzar los propósitos planteados: en primer lugar, se logró estrechar la comunicación entre la familia, los

Es posible ampliar este enunciado en la entrevista realizada por Hernán Suárez a Estanislao Zuleta, disponible en la revista Educación y democracia, p. 19. 
estudiantes y el currículo, a través de la creación del canal virtual Gabrielito Piloso y de distintos blogs, que, al tiempo, funcionan como dispositivos de interlocución que conectan las prácticas de lectura y escritura a la vida cotidiana del estudiante, padres de familia y del aula $^{6}$; cada link se abre como un libro a nuestro aprendizaje, convirtiéndose en centro de acopio de las historias de vida, emociones y crecimiento de quienes hemos construido la línea histórica del tiempo de Apapaches; en segundo lugar, fue posible crear didácticas centradas en el juego, que han permitido a los estudiantes desarrollar habilidades de expresión oral y escrita mientras gozan y disfrutan de la lectura.
La experiencia Apapaches ha permitido el crecimiento en la autoestima de los alumnos y la movilización de la institución educativa hacia prácticas más participativas y significativas de lectura, escritura y oralidad, mientras, al tiempo, propicia el tránsito pedagógico de un aprendizaje memorístico y repetitivo a un aprendizaje mediado por el aspecto social y cultural del estudiante.

A continuación se presenta una expresión gráfica que da cuenta de la estrategia.

\section{Gráfico 2. Estrategia desarrollada en el proyecto Apapaches}

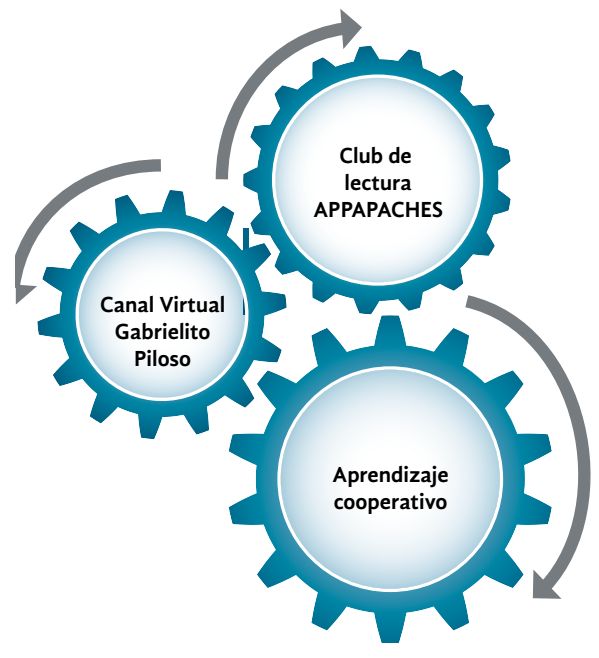

Así, en el aula están presentes la comunicación, la creatividad y los desafíos, elementos de interacción que provocan y entusiasman, mientras funcionan como principios integradores del aprendizaje cooperativo (trabajo en equipo, intercambio de saberes, producción de conocimiento) y como estructura de Apapaches desde cuatro momentos en la actividad escolar: intencionalidad, construcción, participación y transformación.

Se recomienda consultar al respecto: http://consentidoprimerainfancia. blogspot.com.co/ 
Imagen 1. Tipología ilustración, desde las temáticas de los proyectos de aula. Nuestro planeta Tierra, Gabito en mi colegio
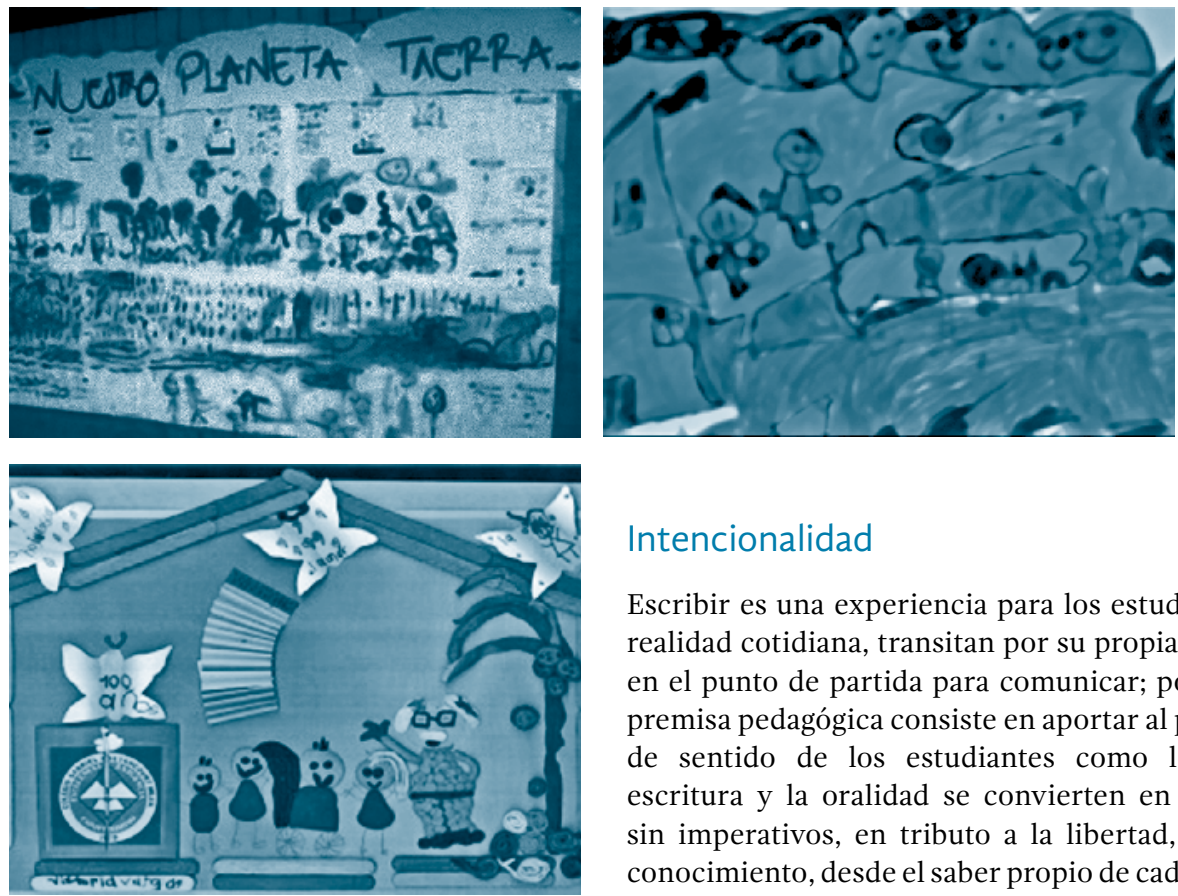

\section{Intencionalidad}

Escribir es una experiencia para los estudiantes, hace parte de su realidad cotidiana, transitan por su propia historia, convirtiéndola en el punto de partida para comunicar; por ello, en Apapaches la premisa pedagógica consiste en aportar al proceso de construcción de sentido de los estudiantes como lectores. La lectura, la escritura y la oralidad se convierten en actividades académicas sin imperativos, en tributo a la libertad, la alegría, el goce y el conocimiento, desde el saber propio de cada estudiante. El ejercicio de escritura literaria se construye en un constante ir y venir hacia y desde la literatura; por ejemplo, en la creación de historias de cada uno de los estudiantes cuando, con su experiencia en Apapaches, se sumergió, desde su propio interés y conocimiento, en los diferentes temas propuestos en el concurso Leer y escribir de la Secretaría de Educación de Bogotá; creando sentidos, enlaces culturales e historias genuinas.

Desde muy pequeña he estado muy interesada en los libros y todo lo que tenga que ver con Literatura. Cuando supe sobre la Feria del Libro deseé asistir a todas las ferias. A mis seis años de edad [...] había un grupo de lectura en el cole, el club de lectura Apapaches [...] Me han dicho muchas veces que después de leer un libro uno es diferente, uno construye diferentes pensamientos y diferentes formas de ver la vida (Adriana Lucía Bravo, grado noveno, segundo puesto, reseña, Bogotá: de feria en feria, 2016). 
Imagen 2. Salida a la Feria del Libro de Bogotá 2011- 2012
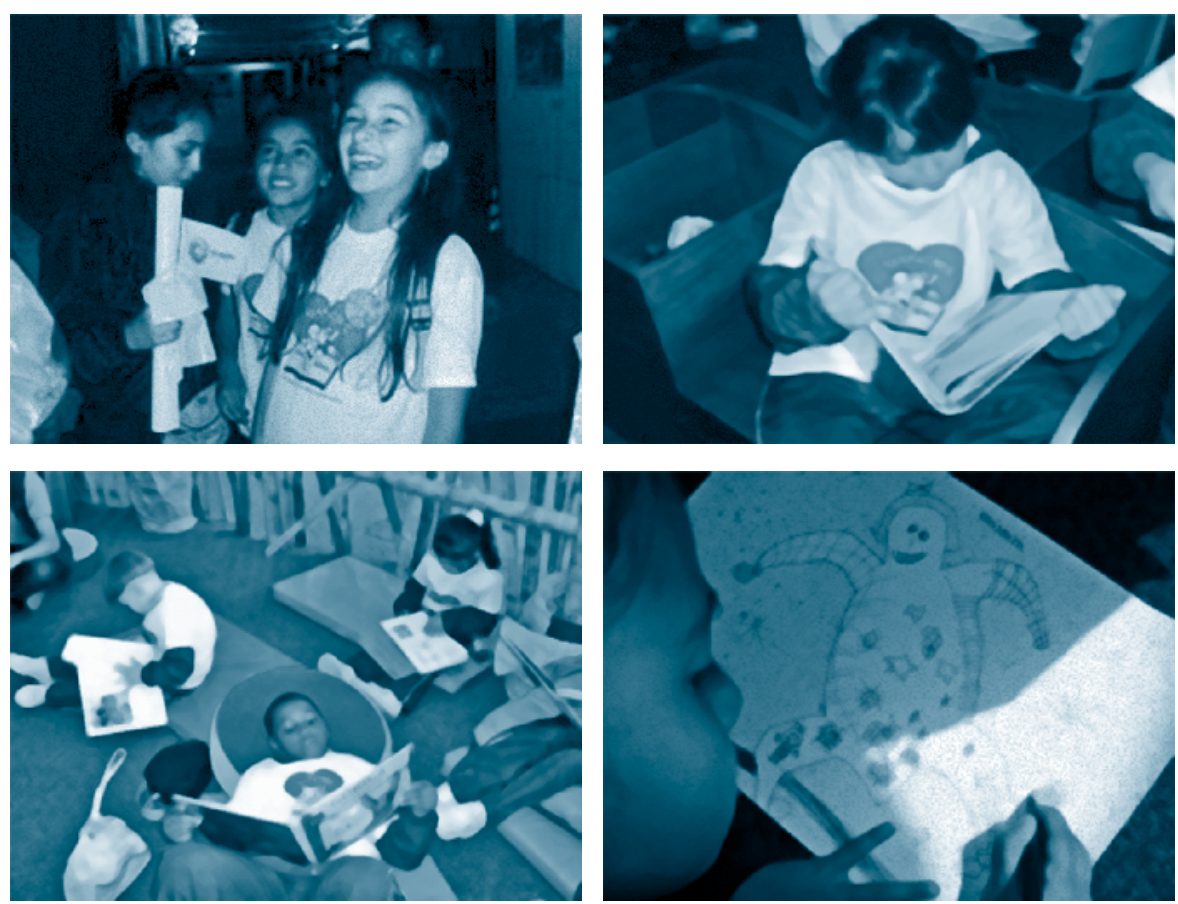

Imagen 3. Concurso Orden al Mérito Literario Don Quijote de la Mancha 2017-2018
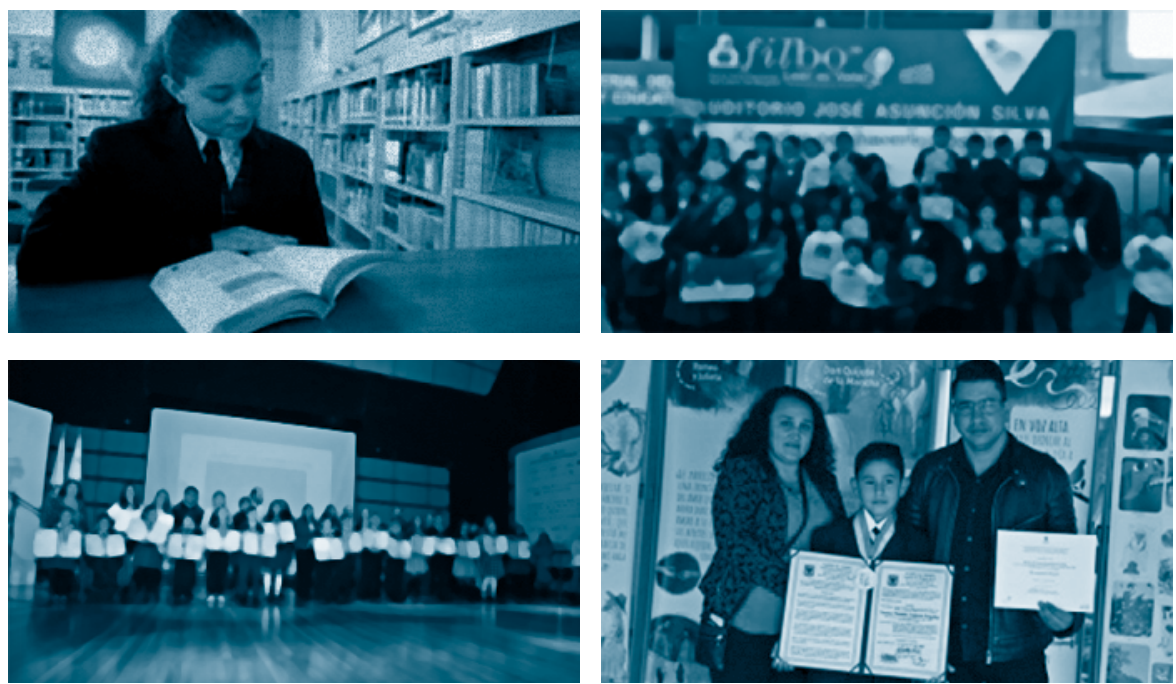

Cuando tenía siete años, mi primera profesora, una persona muy especial, me inculcó el amor por la música, el arte y la cultura [...] De pequeña siempre me imaginé interpretando instrumentos de cuerda como el violín [...] Como en la magia del preescolar, funcionó la varita mágica al concederme el mayor de mis deseos y dar inicio a mi encuentro con la música [...] Esta ha sido la experiencia más maravillosa de mi vida. Cada sonido, vibra y compás son la melodía de mi vida (Jennyfer Tatiana Flórez Delgado, grado noveno, primer puesto, reseña, Notas de juventud, 2016). 


\section{Construcción}

Escribir correctamente es una tarea psicológicamente compleja, por ello, en Apapaches los ambientes de aprendizaje para la escritura se nutren y acompañan desde tres sub-procesos planteados en los modelos cognitivos, los cuales no se dan de una manera lineal, sino que van y vienen:

\section{Sub-proceso de planeación}

Se trata de una proyección del texto: ¿Para quién escribo?; ¿qué quiero comunicar?; ¿a quién va dirigido?, preguntas que remiten directamente al tipo de audiencia y conectan con los posibles lectores; por ejemplo, esto sucede, en el caso de Apapaches, con Samuel Esteban Carranza Caballero, del curso segundo, quien ganó el primer puesto en la categoría de cuento, con su relato "Samuel en Trasmilenio" ", que inicia con la pregunta ¿Qué le gusta a Samuel de Bogotá?, planteando como respuesta: montar en Transmilenio junto a su familia y mirar por la ventana. Mientras que Julián David Jaimes Cárdenas, del grado jardín, segundo puesto en la categoría de ilustración, hizo lo propio en su obra "Mis amigos y yo en Trasmilenio".

Solo la imaginación de un niño y la literatura pueden hacer mágica una situación cotidiana y caótica como el viaje en el transporte público bogotano. Para expresarse, los niños deben poder razonar sobre cosas que conocen directamente, que forman parte de su vida. No pueden dar su punto de vista sobre historias lejanas o países y problemas que no conocen, pero sí de la vida del barrio, de la ciudad en que viven, de sus necesidades y deseos. Es importante incluirlos, comprometerlos con problemas que tocan la opinión de todos (Tonucci, 2002, p. 21).

\section{Imagen 3. Prácticas de escritura en el aula alrededor del tema para el concurso; Bogotá una historia esperando ser contada; Samuel en trasmilenio}
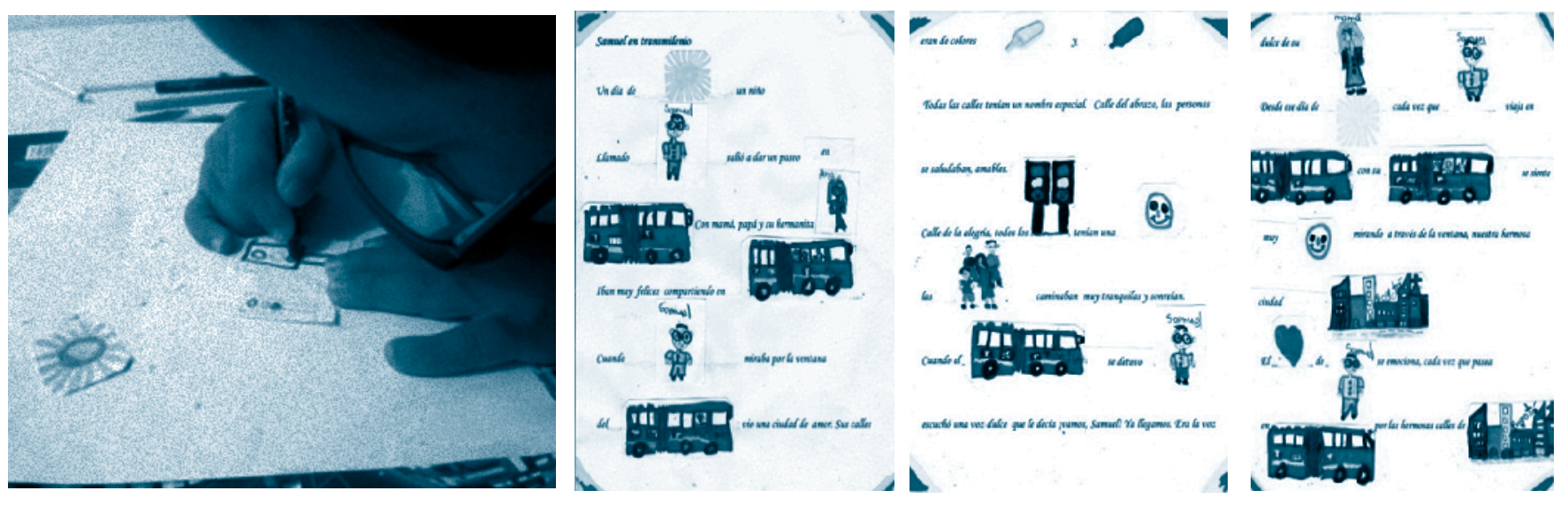

\footnotetext{
Es posible consultar en http://www.educacionbogota.edu.co/es/sitiosde-interes/nuestros-sitios/agencia-de-medios/noticias-intitucionales/ os-pequenos-grandes-escritores-de-los-colegios-de-bogota-se-tomanla-filbo-2017
} 


\section{Sub-proceso de transcripción/traducción}

Cada idea es importante y va conectada a la intención comunicativa, al pensamiento, a su propio lenguaje; así, en Apapaches el estudiante comienza por cuestionarse y encontrar su propio estilo, paso a paso va encontrando la mejor versión de su escrito.

\section{Sub-proceso de revisión/edición}

En Apapaches la escritura siempre cuenta con una audiencia lectora, esto implica un mayor compromiso del estudiante para pulir su texto y reconocerse como escritor; en los cursos de jardín y transición su actividad intelectual en este proceso gira alrededor de comparar la forma de sus grafías con la de las letras del alfabeto, hecho que le lleva a conversar con los amigos de curso, contrastar, preguntar, actuar y decidir sobre su texto.

\section{Participación, oportunidad de encuentro con el público lector}

En Apapaches los escritos tienen una intención comunicativa dirigida a una audiencia; el momento de participación gira alrededor de la interacción de los estudiantes con diferentes escenarios, tales como exposiciones frente a los padres de familia y la intervención en concursos institucionales, distritales, foros educativos, foros de aula o la presentación de sus textos en círculos de lectura compartida; los jurados son en ocasiones sus propios compañeros o docentes de otros cursos y también consideran los comentarios realizados en la red. La actividad intelectual de los estudiantes de jardín y transición consiste en diferenciar entre dibujo y escritura. Las rúbricas o rejillas de evaluación se convierten en la voz del auditorio que dialoga con el estudiante, permitiéndole conocer su nivel y plantearse nuevos retos en su proceso lector y de escritura.

Imagen 4. Actividad de preparación para el concurso. Ilustraciones, Mis amigos y yo en trasmilenio, Remedios y

Gabito visitaron mi ciudad educadora, una mariposa juguetona en Macondo, jugando a la ronda con Gabito.
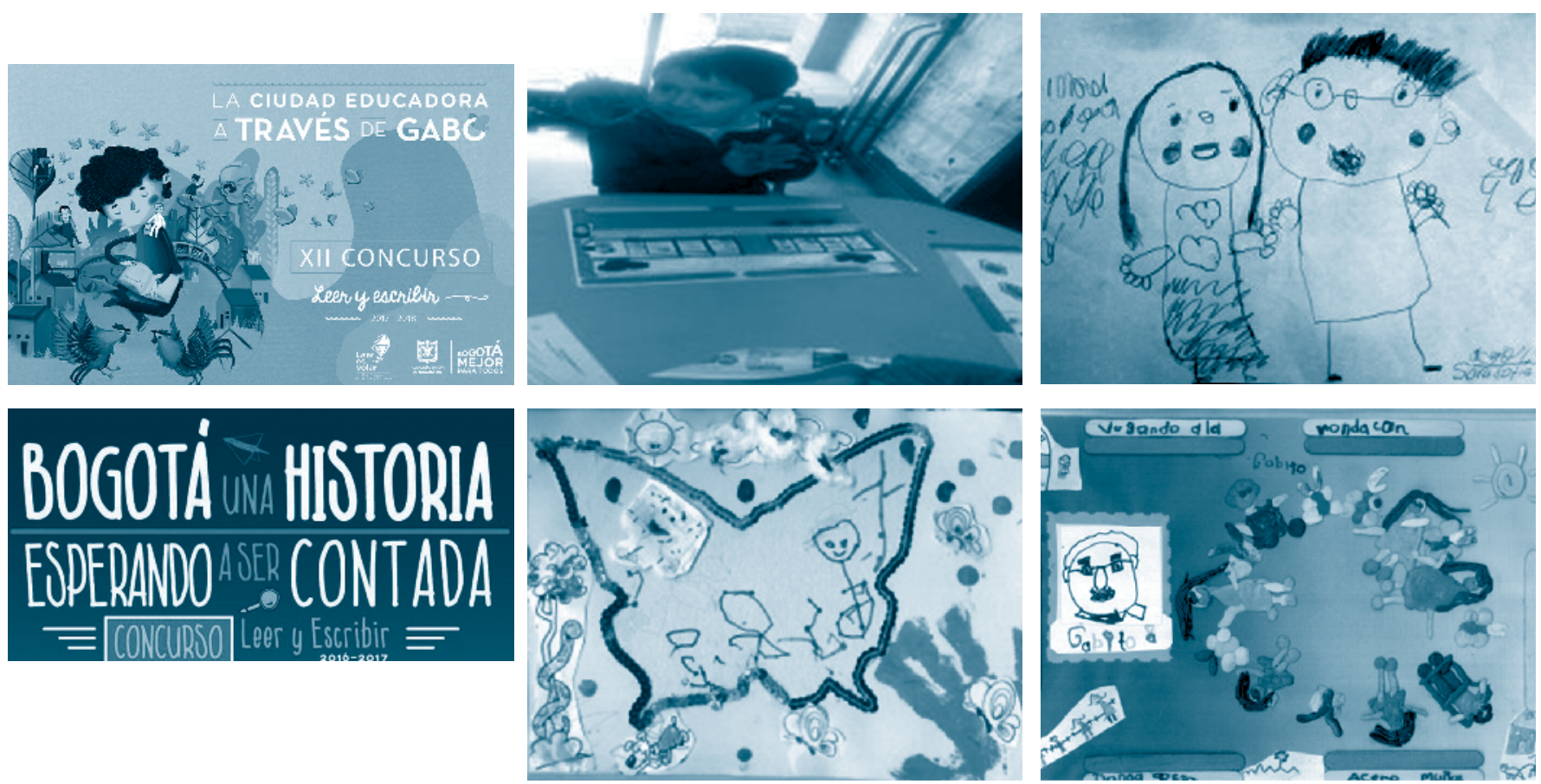
Al tiempo, leer y escribir son un gusto que se transmite en doble vía: de maestro a estudiante y de estudiante a maestro. En Apapaches la docente comparte sus pasiones y saberes; de este ejercicio nace "Pacha la cuenta cuentos", actividad que ha llevado a la profesora a convertirse en mediadora en la lectura y narración de cuentos. Cada encuentro es entonces un regreso a sus experiencias, a casa, al regazo de la madre, leyendo un libro o esperando los domingos para encontrar las tiras cómicas del Magazín Dominical: Mafalda,
Benitín y Eneas, Lorenzo y Pepita, Pancho y Ramona, Calvin y Hobbes, Fernan'd e incluso Olafo el Amargado; volviendo a la radio y a personajes como Kaliman, junto a su inseparable amigo Solín, que alimentaron la capacidad de imaginar. Tal reconstrucción de los hechos sociales y culturales, la propia experiencia de lectura durante la infancia, los símbolos como el cartero, la estampilla y el periódico funcionam a manera de eco que repercute en las decisiones que se toman en el aula.

Imagen 5. Actividad de lectura en voz alta. Pacha, la cuenta cuentos y dinamizadores de lectura, visita a la biblioteca pública, El Tintal.
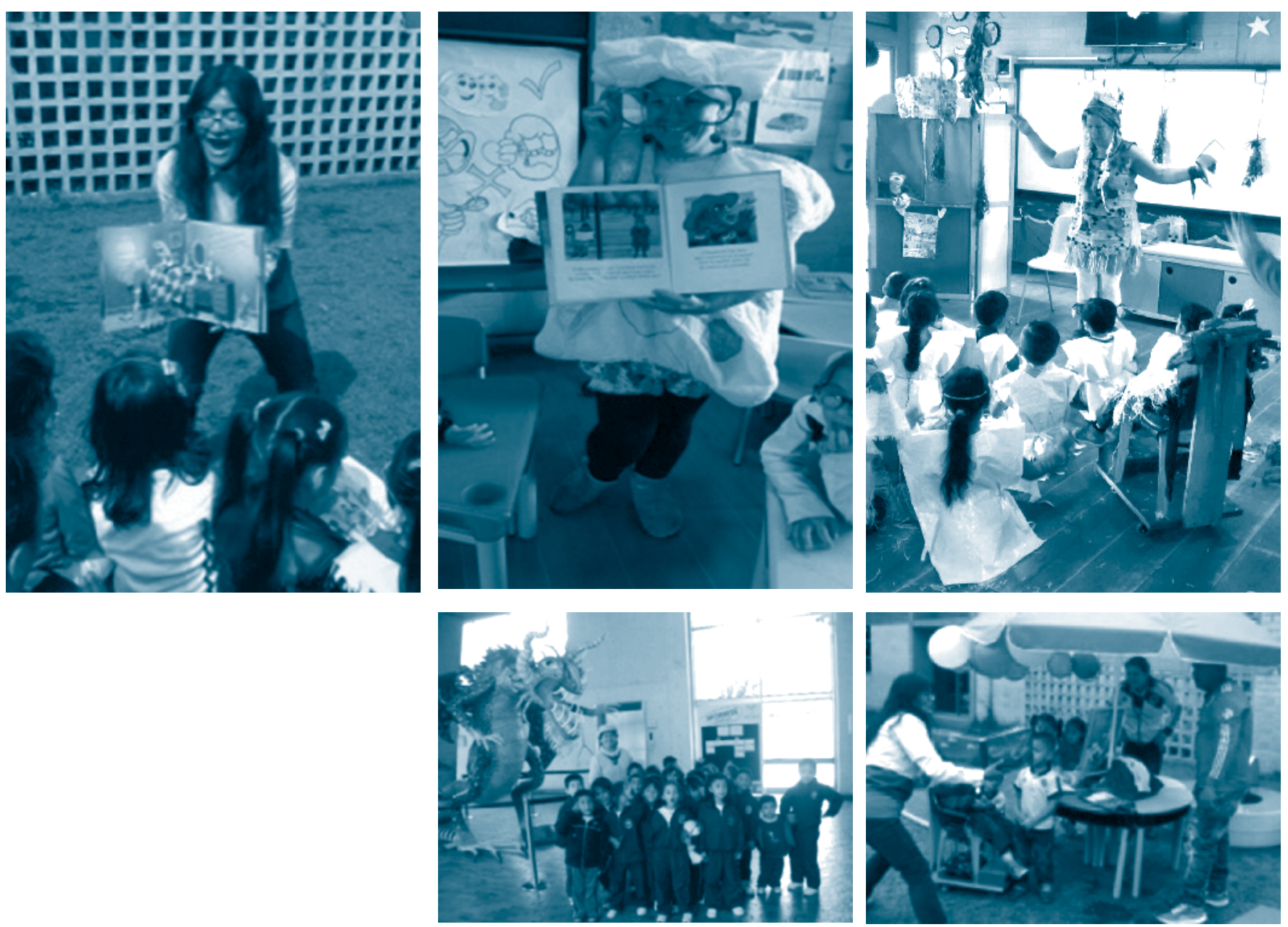


\section{Transformación}

El producto escrito se acompaña y construye en clase, junto a los docentes y amigos de curso; se socializa en casa y se lee en compañía de los padres. No existe la tarea del tipo "Haga un cuento y lo trae la próxima clase"; en Apapaches el interés escritor y lector se desarrolla mediado por el afecto y la compañía permanente del maestro. En tal contexto, la Zona de Desarrollo Proximal (ZDP) hace referencia a la distancia entre el desarrollo actual y el potencial; el primero, el momento en el cual el niño resuelve problemas de forma independiente, el segundo, la fase en la cual la solución de problemas se realiza con ayuda de un adulto guía o de un niño con mayores conocimientos (Vygotsky, citado por Flórez, 2004, p. 200).

De la misma forma, Apapaches incluye distintas formas de apropiarse del entorno, tales como la creación del "Menú literario", el paseo de la maleta viajera por los escenarios escolares o la creación de "Pacha la cuenta cuentos". Recursos que entusiasman a los estudiantes por la lectura y facilitan el rol docente como mediador. Las salidas pedagógicas con los estudiantes a cada una de las ferias del libro de Bogotá también se han convertido en la inspiración perfecta para seguir "afinando la pluma" de nuestros jóvenes escritores de "Apapaches".

Tabla 1. Diferencias entre Apapaches y la enseñanza clásica

\begin{tabular}{|c|c|}
\hline $\begin{array}{c}\text { Prácticas } \\
\text { tradicionales para } \\
\text { la enseñanza de la } \\
\text { lecto-escritura }\end{array}$ & $\begin{array}{l}\text { Apapaches: Micro política } \\
\text { educativa en el aula, en el marco de } \\
\text { la felicidad y la expresión }\end{array}$ \\
\hline $\begin{array}{l}\text { Copia } \\
\text { Transcripción } \\
\text { Silencio } \\
\text { Disciplina } \\
\text { Homogenización } \\
\text { Calificación } \\
\text { Censura al error }\end{array}$ & $\begin{array}{l}\text { Currículo flexible } \\
\text { Bazares literarios } \\
\text { Libretas personalizadas } \\
\text { Menús literarios } \\
\text { Cartillas musicales } \\
\text { Salidas pedagógicas } \\
\text { Ambientes de aprendizaje } \\
\text { Campo virtual, juego dramático, entorno } \\
\text { Publicaciones escritas (imprenta escolar) }\end{array}$ \\
\hline
\end{tabular}

\section{Escenarios. Ambientes de aprendizaje cooperativo}

\section{Juego dramático}

Se trata de un ambiente centrado en ejercicios de comprensión desde la oralidad, que funciona como primer escenario de aprendizaje en Apapaches, partiendo de las ideas de Vigostky, desde la perspectiva de entender el juego simbólico de los niños como un complejo sistema del lenguaje. Así construimos, de manera colectiva con los estudiantes de preescolar, "guiones dramáticos", integrando la literatura, la convivencia y el aprendizaje de la lectura y la escritura. Dicho escenario permite que la lectura en voz alta, empleada como herramienta pedagógica, nutra los imaginarios de niños y niñas y despierte el sentido de escucha, mientras la capacidad de ejercer imaginación les facilita la creación de una nueva relación entre el campo del significado y el visual.

\section{Las tecnologías}

Son empleadas como recurso para optimizar y precisar la comunicación entre familia, estudiantes y currículo. El proyecto cuenta con un canal: Gabrielito Piloso, auspiciado por Apapaches y la Institución Educativa Gabriel Betancourt Mejía, desde su programa con énfasis en TIC. Cada pieza audiovisual del canal funciona a partir de la conjunción entre la intención pedagógica (IP) y la intención comunicativa de la imagen (IC); así, como docente, me es posible una transformación en directora de escena con el sonido de la claqueta, por ejemplo, la intención pedagógica de aprender a escribir el propio nombre, combinada con la intención comunicativa de la imagen, permitió crear un producto ${ }^{8}$ en el cual los elementos identitarios hacen parte de escribir el nombre, teniendo como cuerpo de consulta el contexto escolar, conformado por los amigos de curso, quienes permiten disfrutar de una experiencia estética de la lectura y la escritura al sostener comunicación fluida con los pares. Antes que "un" texto, las interfaces gráficas componen una pluralidad de textualidades entrelazadas.

Al tiempo, vale la pena señalar una segunda experiencia relevante: Enseñanza de mitos y leyendas Ícaro, que comienza con la intención pedagógica y se combina con la intención comunicativa de la imagen en el siguiente producto: https://youtu.be/Lac4x3tYSz8. Aquí la Literatura se transforma en un arte de jugar con la palabra, instalándose en la escena humana para hacerla propia y concibiendo a los niños como sujetos dotados de razón, de vida consiente de sí misma (Fromm, 2007).

El ambiente tecnológico también incorpora a los padres de familia, desde sus propios ritmos y tiempos; tareas como escribir comentarios en cada página les une a sus hijos en una actividad de lectura y escritura para comunicar emociones 9 . Compartir espacios en familia significa llevar a los estudiantes hacia otros esquemas de comunicación, distintos de los habituales como la T.V. Las narrativas construidas por los padres y madres son productos comunicativos que expresan su identidad, pero a la vez otorgan la oportunidad de construir identidad a partir de narrativas.

\footnotetext{
8. Es posible consultar el trabajo en: https://youtu.be/FuvfvV2SmNU y en https://youtu.be/ NpgGn5pOnQU

Se recomienda consultar: https://youtu.be/r2xLCjlsflo
} 
Por otra parte, en Apapaches la gramática del dibujo ${ }^{10}$ se ha constituido a partir de la conexión entre aprendizaje y evaluación, un texto que puede ser leído sin palabras por la docente. Se trata de una comunicación no verbal que ilustra la forma de expresión y conocimiento que habita en el mundo de cada uno de los estudiantes. Como nota personal, colecciono sus dibujos con la misma emoción que, de pequeña, recolectaba las "monas" para mi álbum "Amor es"; prestar atención a sus dibujos me ha permitido evaluar las diferentes dimensiones de su desarrollo: la personal social, la cognitiva, artística, corporal y comunicativa.

\section{Bazar Literario Gabrielista. Espacio de interacción literaria}

Desde el año 2013 Apapaches ha concretado distintas publicaciones estudiantiles, entre ellas, el Primer Concurso de Ilustración Gabrielista, en el que construimos el cuento "La cebra presumida", en el año 2017 la producción literaria "De Apapaches por Bogotá" de los estudiantes participantes del concurso leer y escribir de la SED, Bogotá una historia esperando ser contada, guiados por la idea de que el sentido democrático de la literatura no radica en que todos seamos escritores, sino en el hecho de que aprendamos desde la infancia a disfrutar de la palabra escrita (Rodari, 2003).

Con ello en mente, la propuesta avanza en la tarea de llevar a los estudiantes a conquistar espacios alternos al aula. Conectar el canal Gabrielito Piloso al contexto escolar ha permitido establecer una comunicación mediada por el computador o el celular, lo cual implica, para padres, niños y niñas, integrar en un solo momento lectura, escritura, socialización, imagen y texto; sin contar con la expectativa de entusiasmo que les produce encontrar sus dibujos, familia, amigos y textos, y apreciar sus producciones a través de la pantalla.

Imagen 6. La ilustración como actividad de comprensión y construcción de significado

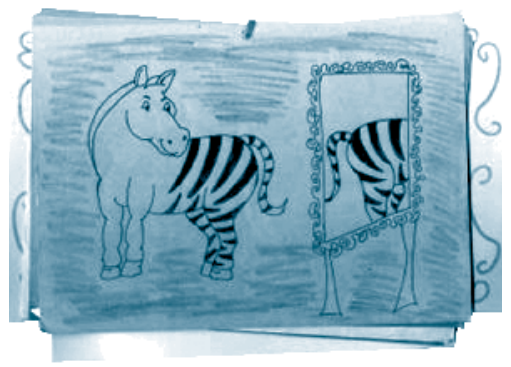

Nota. Autora: Erika Mahecha, año 2013

10. Como ejemplo de este tipo de trabajo es posible revisar: https://youtu. be/RGSaassrdeM
El voz a voz virtual de Gabrielito Piloso también ha llegado a las familias de los estudiantes diagnosticados con Síndrome de Down, Parálisis Cerebral, Hemiplejia y Mielomeningocele del Colegio Gabriel Betancourt Mejía; confirmando el carácter inclusivo de la propuesta, en consonancia con el enfoque diferencial, que invita a maestras y maestros a profundizar en la comprensión de las niñas y niños como sujetos diferenciados y protagonistas de su desarrollo, así como de sus modos de comunicación.

Imagen 7. Actividad de lectura; escritores al alma

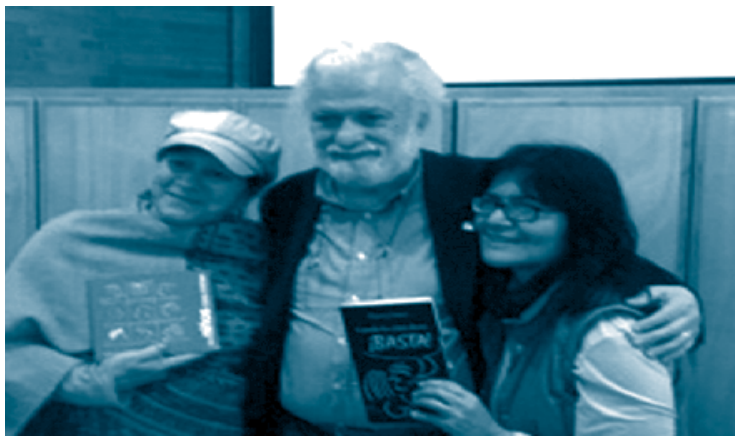

Franceso Tonnucci Yolanda Reyes

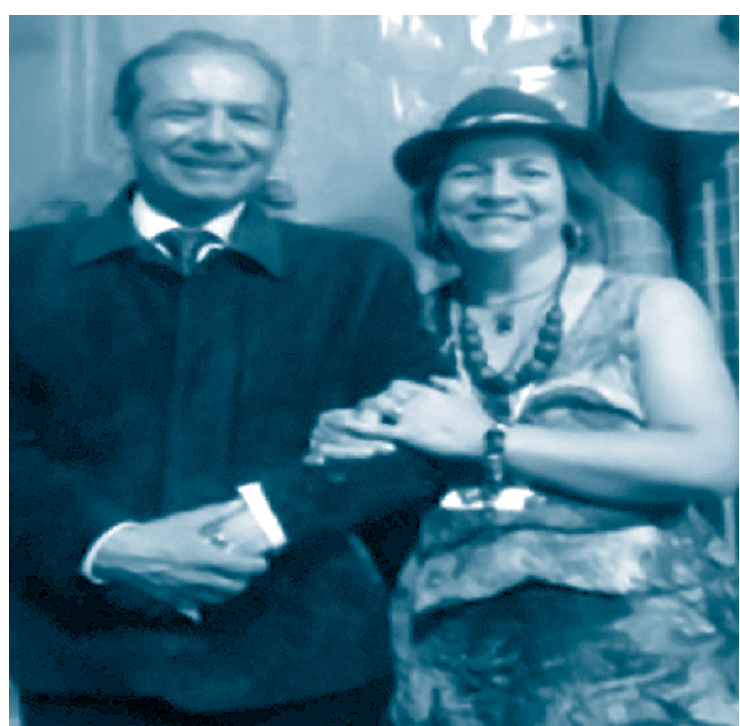

Celso Román

190 EduCACIÓN y CIUDAD NNo 34 ENERo - JUNIO de 2018 ISSN 0123-425 WeB-ONLINE 2357-6286 PP. 177-192 

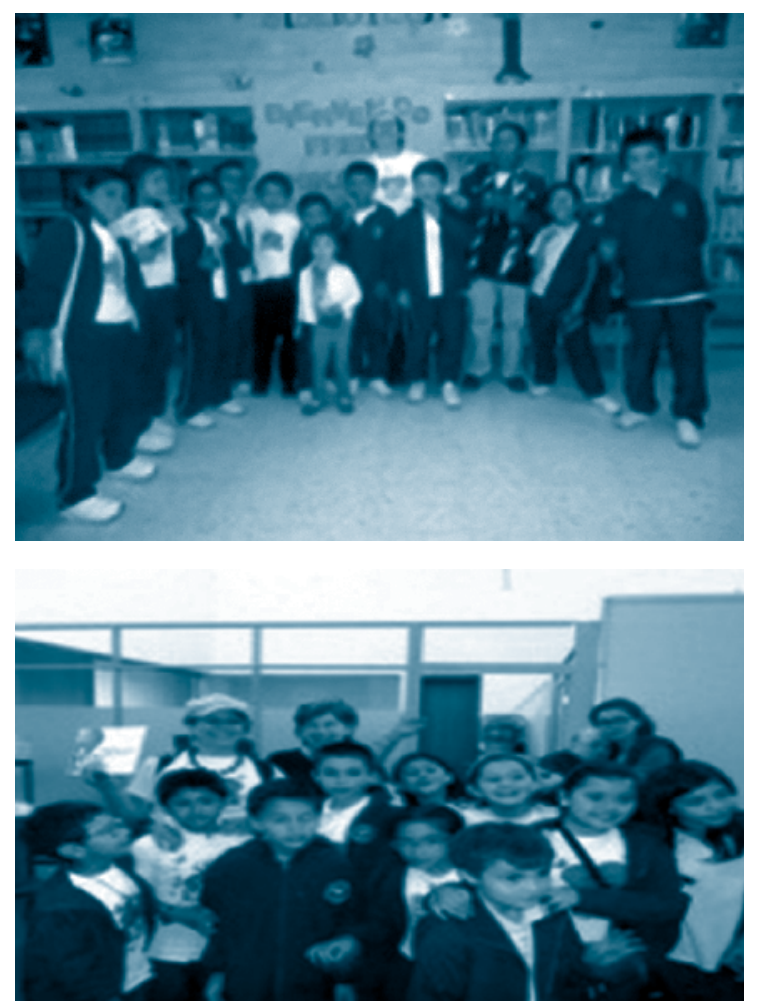

Fredy Chikangana

\section{El entorno: Ambiente de aprendizaje dotado de multiculturalidad a la espera de ser explorado y apropiado por los estudiantes}

La Biblioteca Pública El Tintal, Manuel Zapata Olivella, cercana a la Institución Educativa Gabriel Betancourt Mejía es, parafraseando a Borges: "lo más cercano al paraíso"; en ella, y en las bibliotecas públicas de Biblored, he tenido el privilegio de disfrutar junto a mis estudiantes y mi hijo, Mateo, el placer exquisito que conecta al hombre con la palabra escrita y al conocimiento con el acto de leer. De esta pasión por los libros nace el emblema de nuestro proyecto: Apapaches. En este espacio leer es acariciar el alma con los libros.

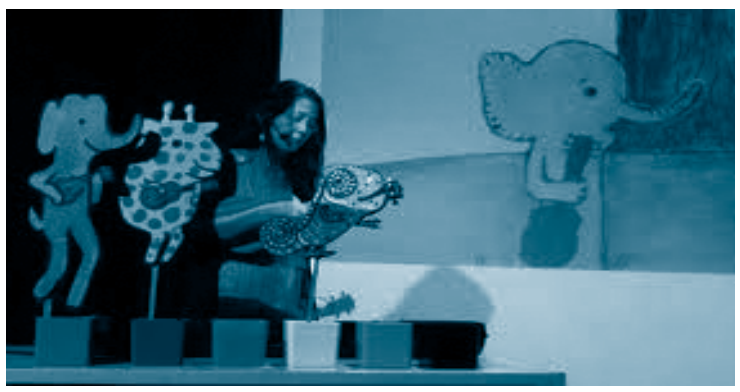

Amalia Low

\section{Reflexiones finales}

Apapaches moviliza las prácticas de aula para el aprendizaje de la lectura, escritura y oralidad, asumiendo el reto de afrontar sin miedo ni engaños las prácticas tradicionales que han habitado por tanto tiempo el contexto escolar, mutilando la creatividad, la confianza y el desarrollo integral de nuestros estudiantes. Por mucho tiempo se promulgó un currículo único e inamovible, es el momento de salir con nuestros estudiantes al encuentro con la diversidad, la felicidad, la multiculturalidad.

Los esfuerzos y avances del Ministerio de Educación Nacional y de la Secretaría de Educación Distrital serán letra muerta si no se transforman, desde la base, las didácticas escolares, llevándolas a ser instrumentos que aporten en la construcción de un diálogo efectivo entre el estudiante, la familia y el currículo, considerándolos desde sus características propias.

El ejercicio de plantear una micro política de educación en el aula para el aprendizaje de la lectura, la escritura y la oralidad, enmarcado en la felicidad y la expresión, implica entender que las didácticas alternativas, llevadas al aula, contribuyen al intercambio de saberes, a la comunicación de doble vía (contexto-escuela) y a un currículo flexible que adquiere la forma y características propias de cada contexto para, desde allí, actuar y "hacer” educación. 


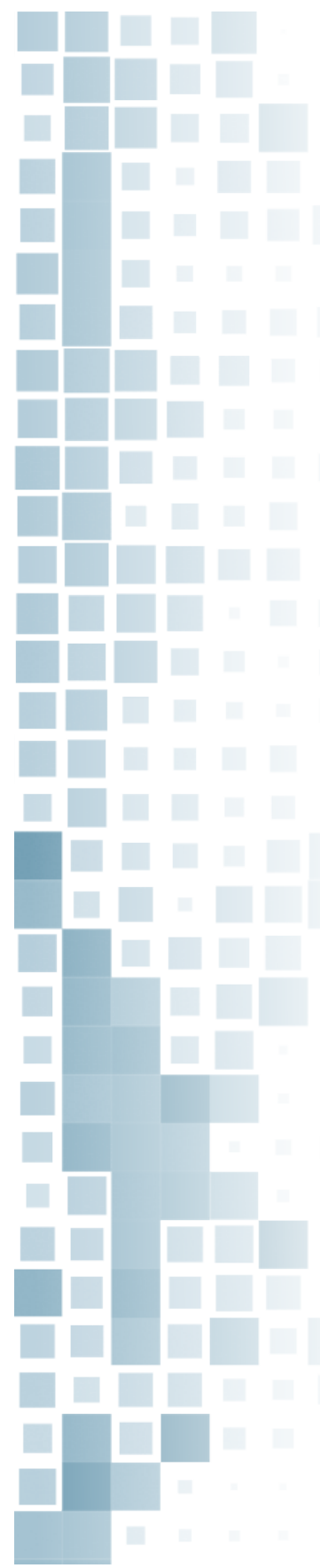

\section{Referencias}

Avendaño, F., y Perrone, A. (2012). El aula: un espacio para aprender a decir y a escuchar. Buenos Aires: Homo Sapiens.

Borges, J. L. (1998). Borges oral. Madrid: Alianza Editorial.

Brunner, J. (1999). Actos de significado, más allá de la revolución cognitiva. Madrid: Alianza:.

Flórez R., R. (2004). El lenguaje en la educación, una perspectiva fonoaudiológica. Bogotá: Universidad Nacional de Colombia.

Fromm, E. (2007). El arte de amar. México: Editorial Harper.

Paronzini, P. (2012). Los niños y las artes visuales en el jardín y la escuela primaria. Buenos Aires: Homo Sapiens.

Rodari, G. (2003). La escuela de la fantasía. Madrid: Editorial popular.

Saussure, F. (1916). Curso de Lingüística General. Buenos Aires: Losada.

Secretaría de Educación del Distrito, SED. (2007). Orientaciones curriculares para el campo de la comunicación, arte y expresión. Bogotá: SED.

Suarez, H. (2010). La educación, un campo de combate. Educación y democracia: un campo de combate, pp. 9-47. Obtenido desde https://omegalfa.es/downloadfile.php?file=libros/educacion-ydemocracia.pdf

Tonucci, F. (2002). Cuando los niños dicen: ¡Basta! Buenos Aires: Losada.

Wertsch, J. V. (1998). Vygotsky y la formación social de la mente. México: Paidós. 